\title{
SUR LE DIAGNOSTIC DU KALA-AZAR
}

\section{Par J. FRAGA DE AZEVEDo}

Si nous voulons apprécier le mouvement statistique des cas de Kala-azar au Portugal, nous ne pouvons le faire avec rigueur qu’à partir de 1950, car ce n'est qu'à cette date que cette affection a été considérée comme maladie à déclaration obligatoire.

Cependant, il m'a été possible de réunir les données relatives aux cas de Kala-azar enregistrés, de 1940 à 1950, dans les hôpitaux de Lisbonne, Porto et Coïmbra, autrement dit dans les Services cliniques les plus importants du pays, ainsi que dans les Services antipaludéens (1), et e'est pourquoi ces chiffres peuvent nous donner également une idée de l'évolution de l'endémie au cours de la période en question. En vérité, si nous comparons le nombre de cas enregistrés à la Direction Générale de Santé en 1950, 1951 et $1952(2,3)$, avec ceux qui ont été enregistrés dans la statistique que j'ai pu élaborer, on constate que les chiffres sont presque superposables pour les années 1951 et 1952 ; cependant, en 1950, la différence est appréciable, comme le montre le tableau I.

TABLfay 1

Cas de Kala-Azar enregistrès au Portugal de 1950 à 1953

\begin{tabular}{|c|c|c|c|c|}
\hline \multirow[t]{2}{*}{ AnNées } & \multirow{2}{*}{$\begin{array}{l}\text { DiRECTION } \\
\text { GÉNÉrALE } \\
\text { DE SANTÉ }\end{array}$} & \multirow{2}{*}{$\begin{array}{l}\text { Hôpitaux de Lisbonne, } \\
\text { Porto, CoÏmbra et } \\
\text { Services dNTipaludés }\end{array}$} & \multicolumn{2}{|c|}{$\begin{array}{c}\text { DIFFÉRENCE EN MOINS } \\
\text { DU 2e GROUPE }\end{array}$} \\
\hline & & & En chiffres absolus & $\%$ \\
\hline 1950 & 448 & 340 & 108 & 32 \\
\hline 1951 & 230 & 190 & 40 & 21 \\
\hline 1952 & 194 & 185 & 11 & 5 \\
\hline
\end{tabular}

Dans ces conditions, en groupant les éléments des deux provenances, nous pouvons suivre l'évolution de l'endémie au Portugal de 1940 à 1958 . 
Si nous traduisons graphiquement les données en question (fig. 1), nous constatons que le Kala-azar a atteint le point le plus élevé d'incidence en 1950 , pour baisser à partir de 1951 , et se maintenir, avec de légères oscillations, à un niveau plus ou moins stationnaire à partir de cette année.

En vérité, la détermination de la ligne de tendance, relative aux neuf premières années à partir de la date où a été établie la déclaration obligatoire de la maladie au Portugal, nous montre que cette ligne (déterminée par le $\mathrm{D}^{r}$ Santos Reis, à qui j'exprime ici mes remerciements) se présente sous la forme d'une courbe parabolique

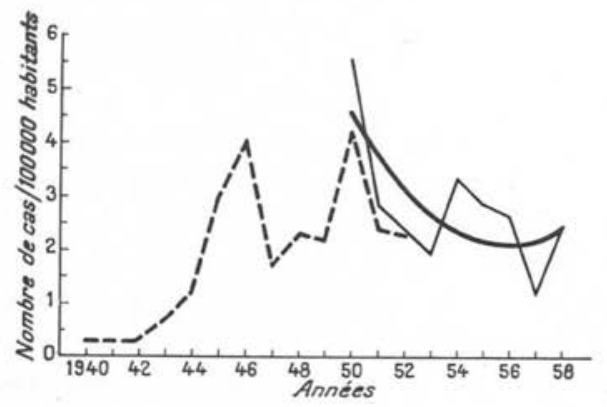

Fig. 1. - Cas de Kala-azar enregistré au Portugal de 1940 à 1958 (ce n'est qu'à partir de 1950 que l'affection a été considérée comme maladie à déclaration obligatoire). La ligne de tendance a été aimablement déterminée par le $\mathrm{D}^{\text {r }}$ Santo Reis.

_ - cas enregistrés dans les hôpitaux de Lisbonne, Porto et Coïmbra et dans les services antipaludéens :

- ligne de tendance.

suave (fig. 1). Cette courbe a été certainement influencée par la hausse d'incidence relativement notable constatée en 1950, car sans cela elle serait certainement plus douce, peut-être presque horizontale, révélant, pour ainsi dire, un ètat stationnaire de l'endémie.

Il est évident que le nombre d'années sur lequel sont fondés ces calculs est très limité, car il se rapporte seulement à la période 1950-1958, et c'est pourquoi les conclusions doivent être interprétées sous toute réserve.

Nous ignorons ce qui se passe actuellement dans le pays quant aux hôtes naturels des Leishmanies, les chiens, car, depuis 1953 (4). on n'a procédé à aucune enquête sur ces animaux en vue de vérifier s'il s'est produit chez eux une réduction du taux d'infection par ces Protozoaires, parallèle à celle qui a été enregistrée chez l'homme.

Entre-temps, il semble que, tout au moins jusqu'en 1953, cette baisse ne se soit pas produite, comme le montre le tableau Il, bien que cette affirmation doive être également admise sous toute réserve, car, comme nous l'avons dit, nous ne pouvons nous fonder sur aucune enquête bien organisée. 
TABLEAU II

Taux d'infection des chiens de Lisbonne par les Leishmanies

\begin{tabular}{|c|c|c|}
\hline AUTEUR ET DATE & $\begin{array}{c}\text { NOMBRE DE CHIENS } \\
\text { EXAMINÉS }\end{array}$ & INFECTÉS \% \\
\hline $\begin{array}{c}\text { Alvares et Pereira da Silva, } \\
1910 / 1911(5) \ldots \ldots \ldots \ldots \ldots \ldots\end{array}$ & 416 & 3,1 \\
\hline Correia Mendes, 1936/1938 (6). & 240 & 2,18 \\
\hline $\begin{array}{l}\text { Fraga de } \text { dzevedo et col., } 1947 \\
(7) \ldots \ldots \ldots \ldots \ldots \ldots \ldots\end{array}$ & 137 & 6,5 \\
\hline Silva Leitão (*) 1953 (4) . . . . . & 50 & 100 \\
\hline
\end{tabular}

(*) L'examen a porté sur des chiens malades et porteurs de lésions de la peau des Services de Salubrité de la Municipalité de Lisbonne.

Ce qui se passe au Portugal quant à la réduction des cas de Kalaazar n'est pas totalement en accord avec ce que l'on constate dans la zone méditerranéenne, à laquelle, à ce point de vue, appartient le pays ; en effet, l'étude de l'incidence du Kala-azar, dans les pays de cette région où il m'a été possible d'obtenir quelques éléments, montre que l'on a enregistré une réduction indiscutable de l'incidence de la maladie. Tel est le cas, par exemple, de l'Italie, où Lippi (8) mentionne 583 cas de Kala-azar en 1946 et 50 seulement en 1950, tandis que, pour la forme cutanée, 2.742 cas ont été enregistrés en 1947 et 500 seulement en 1954. De même, en Chine, le nombre de cas dans quatre zones a baissé de 24,8, 16,5, 12,5 et $9,4 \%$ entre 1950 et 1953 , mais à la suite de mesures contre le Kala-azar : traitement en masse de la population et lutte contre les Phlébotomes (9).

On enregistre ainsi, chez nous, une baisse relative des cas de Kala-azar ; mais il faudrait préciser en premier lieu si la réduction constatée correspond à la réalité, ou bien si nous ne traversons pas simplement une période de réduction cyclique naturelle, d'une manière analogue à ce qui se passe dans d'autres régions. C'est ainsi que la maladie accuse aux Indes, pendant 4 à 10 ans, des recrudescences appréciables, suivies de longues périodes d'accalmie tous les 15 ou 20 ans ; il en est de même en certaines régions de Chine, où 
le Kala-azar présente des aspects épidémiologiques analogues à ceux du Kala-azar méditerranéen.

En admettant que cette baisse d'incidence correspond chez nous à un véritable déclin de la maladie, et non à une réduction temporaire, nous pouvons l'attribuer aux facteurs suivants, considérés isolément ou associés :

$1^{\circ}$ Amélioration des conditions générales d'hygiène de la population ;

$2^{\circ}$ Réduction du nombre de chiens errants, hôtes naturels, chez nous, de la Leishmania donovani ;

$3^{\circ}$ Réduction de l'incidence des Phlebotomus comme vecteurs possibles de l'agent de la maladie.

Quant au troisième facteur, celui qui mérite d'être considéré plus attentivement, je rappellerai que, dans de précédents travaux $(1,10)$, j'ai exprimé mes doutes sur le rôle qui est attribué aux Phlebotomus comme vecteurs du Kala-azar, car j'ai eu l'occasion de prouver que l'intervention de ces Insectes n'explique pas, à elle seule, toute l'épidémiologie du Kala-azar méditerranéen.

Je dois souligner que les considérations alors exposées ont obtenu l'accord de quelques auteurs et n'ont pas encore été réfutées, bien qu'elles aient fait également l'objet d'un article publié dans les Archives de l'Institut Pasteur d'Algérie, où a pris naissance l'école qui soutient la transmission des Leishmanies par les Phlebotomus.

Quoi qu'il en soit, il est certain que l'application sur une grande échelle des insecticides à action résiduelle contre les Insectes, et en particulier dans la lutte antipaludéenne, a fait baisser l'incidence du Kala-azar dans les pays où ces mesures ont été appliquées. Il en a été ainsi dans certaines zones de l'Italie (11) où, après l'application du D.D.T., à partir de 1948, le nombre de cas actifs a baissé de $2,9 \%$ en cette même année à $0,04 \%$ en 1953 , tandis que dans les zones témoins, non traitées, les pourcentages ont été de 3,5\% en 1948 et de $4 \%$ en 1950.

Si nous examinons l'évolution du Kala-azar au Portugal en relation avec l'application systématique des insecticides, il semble que se confirme également chez nous l'action salutaire de ces derniers, car, ayant été appliqués contre la malaria à partir de 1949 , on a constaté à partir de 1950 une baisse notable de la maladie. De même, dans le Pakistan Oriental (12), on a commencé à enregistrer une réduction des cas de Kala-azar un an après l'application du D.D.T. contre le paludisme. 
Chez nous, eependant, la réduction des cas de Kala-azar n'a pas été aussi sensible que celle des cas de paludisme, car le nombre de ces derniers a passé de 5.882 en 1950 à 12 en 1958 , dont quatre seulement autochtones (13), tandis que la baisse, comme nous l'avons dit, n'a pas été aussi expressive pour le Kala-azar. Ainsi le montre clairement le graphique logarithmo-arithmétique (fig. 2) de l'évolution des deux maladies dans la période en question, élaboré également par le $\mathrm{D}^{r}$ Santos Reis. Une telle différence dans les résultats peut se justifier soit par le fait, comme je l'ai dit, que les Phlébotomes n'expliquent pas toute l'épidémiologie de la maladie, soit parce qu'en raison de la biologie spéciale des espèces du pays, ils ne sont pas autant affectés que les Anopheles par l'action des insecticides.

Cependant, quant à moi, ce n'est

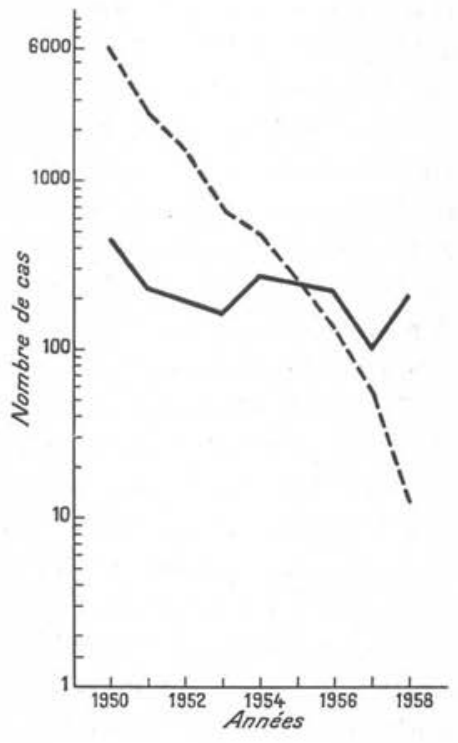

FIG. 2. - Graphique logarithmo-arithmétique de l'évolution du paludisme et du Kala-azar au Portugal, de 1950 à 1958. (Aimablement déterminé par le $\mathrm{D}^{\mathrm{r}}$ Santos Reis). pas seulement l'épidémiologie du Kala-azar qui est enveloppée d'un voile de mystère pour ce qui est de la transmission de la maladie. Le même mystère entoure également d'autres aspects des leishmanioses, comme, par exemple, la question des véritables relations existant entre la forme viscérale et la forme cutanée.

Ainsi, nous ne pouvons manquer d'être surpris par le fait que, bien que le Kala-azar ait été signalé au Portugal depuis 1910, ce n'est qu'en 1943 que l'on a constaté le premier cas de leishmaniose cutanée (14), alors qu'il est certain que dans l'Espagne voisine, appartenant au même bloc géographique, ce type de maladie est enregistré au moins depuis 1936 (15). Il est également étrange que des cas successifs de maladie aient été signalés dernièrement du Nord au Sud du pays (16), totalisant actuellement, à ma connaissance, 11 cas, tout en présumant que d'autres, en nombre chaque fois plus élevé, seront diagnostiqués dans l'avenir.

Une telle constatation de la forme cutanée de la leishmaniose au Portugal soulève de nombreux problèmes, comme, en particulier, ceux qui concernent l'identité des agents responsables de la forme 
viscérale et cutanée, les particularités de dissémination de l'une et de l'autre, et bien d'autres aspects encore obscurs de l'épidémiologie des deux formes, question dans laquelle je n'ose pas m'engager, car elle sort du cadre du présent travail, et parce que je courrais le risque de ne pas aboutir à une conclusion formelle, à tel point le problème se présente encore complexe.

Quels que soient, cependant, les facteurs à invoquer, il est indéniable que nous assistons depuis dix ans à une certaine réduction des cas de Kala-azar dans le pays, ce qui, d'un côté, limite l'importance de la maladie, mais, d'un autre côté, rend son diagnostic plus difficile, étant donné que, par suite de la rareté des cas, les médecins en perdent l'habitude, et ainsi l'hypothèse de son diagnostic se présente à eux plus rarement.

En vérité, les maladies qui ont été jadis fréquentes et qui apparaissent aujourd'hui plus rarement, ainsi que celles qui ne surviennent que sporadiquement, offrent toujours, comme il est évident, de plus grandes difficultés de diagnostic que celles dont l'incidence est plus grande. Nous pouvons citer comme exemple de cette affirmation, en ce qui concerne le premier groupe de maladies, le cas récent du célèbre coureur cycliste italien Coppi. Cet athlète étant tombé subitement malade, le diagnostic de paludisme aigu, si facile à formuler en laboratoire, paludisme que la victime avait contracté en Afrique Occidentale Française, n'a été établi que tardivement, puisque, ayant eu la première crise aiguë fébriie, déjà en Italie, le 28 décembre 1959 , ce n'est que le 2 janvier de l'année courante, à 3 heures du matin, qu'a été établi le diagnostic de fièvre tierce maligne, par les Professeurs Fieschi et Villa, et le malade devait mourir à $8 \mathrm{~h}$. $45 \mathrm{du}$ même jour. Et ce fait est d'autant plus surprenant que tout ceci s'est passé dans un pays qui possède les plus brillantes traditions en ce qui concerne l'étude et la connaissance de cette affection : I'Italie, patrie de Grassi, Bastianelli et Bignani, qui ont prouvé définitivement en 1898 le rôle des Anophèles dans la transmission du paludisme ; de Rafaëlle, dont le nom est lié à la découverte du cycle exo-érythrocytique des hématozoaires du paludisme, et de tant d'autres.

Comme exemple du deuxième groupe de maladies, je peux citer celui que j'ai observé moi-même, quant à l'incidence sporadique dans notre pays de la fascioliase hépatique. Bien qu'il s'agisse d'une parasitose relativement facile à identifier étiologiquement, j'ai pu accompagner des cas, grâce à l'intervention du Prof. F. Fonseca, qui ont donné lieu aux diagnostics les plus divers et les plus étranges (17), avant que n'ait été constatée la véritable étiologie de la maladie. 
Par conséquent, tous les moyens capables non seulement de simplifier les techniques de diagnostic de toute maladie, mais encore de les rendre plus accessibles et de les mettre à la portée de tous, sont de plus en plus justifiés, surtout pour les formes les plus rares, et c'est pourquoi j'ai jugé opportun d'apporter ici le résultat que j'ai obtenu dans le diagnostic du Kala-azar par la recherche des Leishmanies dans la muqueuse nasale et par hémoculture. D'ailleurs, la simplicité et l'innocuité de ces méthodes justifient leur diffusion.

Il est certain que de nombreux progrès ont été réalisés dans l'étude de la pathogénie du Kala-azar et, en conséquence, dans l'étude de la répercussion de la maladie sur l'économie générale, et, donc, des diverses modifications de la structure hématique, dont les altérations constituent ainsi une indication précieuse pour le diagnostic; cependant, nous savons tous que c'est aujourd'hui encore sur la recherche des Leishmanies dans l'organisme que repose le diagnostic de cette maladie.

La recherche des Leishmanies a été classiquement réalisée par ponction splénique au début, et par ponction sternale pour l'examen de la moelle osseuse. Récemment (18), la biopsie hépatique a révélé $66,6 \%$ de diagnostics sur 15 cas de Kala-azar américain. Le pourcentage de cas positifs obtenus par les deux premières méthodes est identique, avec une certaine supériorité, cependant, pour la ponction splénique, puisqu'elle a révélé $96 \%$ de cas positifs par l'examen direct, tandis que l'on obtient $87 \%$ par la recherche dans la moelle osseuse. Toutefois, quelques études, comme par exemple celle de Henderson (19), relative à 300 cas étudiés au Soudan, montrent que la ponction splénique sur laquelle cet auteur a toujours fondé son diagnostic a dû être pratiquée dans quelques cas trois et quatre fois avant de se révéler positive. Moyennant cette répétition, l'auteur a totalisé $100 \%$ de cas positifs.

Cependant, et parce que la leishmaniose viscérale est une maladie générale du système réticulo-endothélial, il faut présumer que les Protozoaires respectifs se trouvent localisés dans les cellules mésenchymateuses (20) des vaisseaux sanguins ou du tissu conjonctif même de n'importe quel point de l'organisme ; d'où l'idée de les rechercher dans les sécrétions nasales et luccales, qui est venue en 1934 à Forkner et $\mathrm{Zia}$, en Chine (21), où ces chercheurs ont travaillé. Et, en vérité, on a trouvé des Leishmanies (22) même dans la peau saine de malades atteints de Kala-azar. Cependant, avant que Forkner et Zia aient recherché les Leishmanies dans les sécrétions en question, Bentley (23) déjà avait examiné, en 1904, l'expec-

Ann. de Parasitologie, T. XXXV, $\mathrm{N}^{\circ} 5-6 .-1960$. 
toration, pour la recherche des mêmes Leishmanies, mais il avait abouti à des résultats négatifs. Après Bentley, c'est Shortt (24) qui, en 1924, a suggéré l'examen de l'expectoration, et c'est à Henderson [cité par Shortt et Swaminath (23)], que revient le mérite, en 1930, d'avoir trouvé des Leishmanies dans la sécrétion nasale d'un malade sur lequel il cherchait des bacilles de la lèpre. Bien qu'il y ait certaines différences épidémiologiques entre le Kala-azar méditerranéen, indien et soudanais, comme le montre le tableau III, il est naturel que la biologie de l'agent causal dans l'organisme soit fondamentalement la même pour les trois types de maladie, tout au moins en ce qui concerne sa localisation dans les différents organes.

Et, dans ces conditions, il était à présumer que l'on pourrait rencontrer des formes de Leishmanies dans la muqueuse nasale en appliquant la même méthode pour le Kala-azar méditerranéen ; et c'est de là que nous est venue l'idée de les rechercher chez nous dans cette partie de l'organisme.

La méthode de recherche des Leishmanies dans la muqueuse nasale ne soulève aucune difficulté et est absolument inoffensive, ce qui est un facteur important à considérer, étant donné le danger relatif que présente la ponction de la rate ou l'inconvénient que présente pour le malade la ponction du sternum ou d'un autre os quelconque pour obtenir de la moelle.

Dans le prélèvement du produit de la muqueuse nasale pour rechercher les Leishmanies, ce qui importe c'est de râcler la muqueuse avec une anse ou curette, de manière à la blesser jusqu'à ce que l'on obtienne du sang, car ce n'est qu'ainsi que nous avons la certitude d'àvoir atteint le chorion où les Leishmanies doivent être surtout localisées.

Etant donné que la pituitaire est riche en tissu érectile, particulièrement sur la face convexe du cornet inférieur, il se peut que la recherche en cet endroit conduise à un plus grand pourcentage, mais nous n'avons pas d'opinion formelle à ce sujet, car nous ne nous sommes pas préoccupés, jusqu'à cette date, de l'endroit précis du prélèvement.

Nous devons dire également que, dans les cas de Kala-azar pour lesquels la recherche dans la muqueuse nasale s'est révélée positive, nous avons eu l'impression qu'il était plus facile de faire saigner la muqueuse au moment du prélèvement que dans les autres cas. Le produit obtenu est étendu sur une lame et coloré par la méthode de Leishman ou une autre identique.

Comme complications légères, il faut mentionner seulement quelques cas d'épistaxis qui cèdent facilement à l'application d'un tampon avec ou sans eau oxygénée. 


\section{TABLEaU III}

\section{Principales differences entre le Kala-azar du type indien du type méditerranéen et du type soudanais}

\begin{tabular}{|c|c|c|c|}
\hline CARACtÉRISTIQUeS & $\begin{array}{c}\text { Kala-azar } \\
\text { du type indien }\end{array}$ & $\begin{array}{c}\text { Kala-azar } \\
\text { du type méditerranéen } \\
\text { (zone méditerranéenne } \\
\text { Chine }(a) \text { et Amérique } \\
\text { du Sud) }\end{array}$ & $\begin{array}{l}\text { Kala-azar } \\
\text { du type } \\
\text { soudanais }(b)\end{array}$ \\
\hline $\begin{array}{c}\text { MaNifestations } \\
\text { CliniQues }\end{array}$ & $\begin{array}{l}\text { (Viscérales } \\
\text { (fréquemment } \\
\text { intestinales) } \\
\text { et cutanées }\end{array}$ & $\begin{array}{c}\text { Viscérales } \\
\text { (rarement intestinales) } \\
\text { et cutanées }\end{array}$ & $\begin{array}{l}\text { Viscérales } \\
\text { fréquemment } \\
\text { intestinales) } \\
\text { et rarement } \\
\text { cutanées }\end{array}$ \\
\hline AgES PLUS ATtEINTS & $\begin{array}{l}\text { Prédominance } \\
\text { parmi les } \\
\text { jeunes adultes } \\
(\text { environ } 60 \%) \\
\text { relativement } \\
\text { fréquent chez } \\
\text { les adultes }\end{array}$ & $\begin{array}{l}\text { Prédominance chez les } \\
\text { enfants de } 1 \text { à } 4 \text { ans } \\
\text { (90\% dans la zone } \\
\text { méditerranéenne et en } \\
\text { Amérique du Sud) ou } \\
\text { chez les enfants d'un âge } \\
\text { plus èlevé (Chine); } \\
\text { rare chez les adultes }\end{array}$ & $\begin{array}{l}\text { Fréquent } \\
\text { chez les } \\
\text { adultes } \\
(66 \%)\end{array}$ \\
\hline Hôte & Homme & Chien & Inconnu $(\boldsymbol{c})$ \\
\hline $\begin{array}{c}\text { CAS MORTELS } \\
\text { (sous l'action } \\
\text { médicamenteuse) } \\
(d)\end{array}$ & $2-9 \%(e)$ & $10-20 \%$ & Environ $25 \%$ \\
\hline
\end{tabular}

(a) Récemment, Wu (25) a signalé deux types de Kala-azar chinois : un en Chine Orientale, identique au type indien, et un autre en Chine du Nord-Ouest, identique au type méditerranéen.

(b) D'après Henderson (1937), à la suite de l'étude de 300 cas (19).

(c) La recherche faite récemment (26) sur divers animaux n'a pas conduit à des conclusions définitives sur ce point.

(d) On comprend bien qu'en dehors de la maladie elle-mème les ressources médicales, l'état de nutrition et d'autres facteurs liés aux conditions de vie des populations doivent influencer les taux de mortalité ; c'est pourquoi les chiffres présentés ne peuvent être considérés qu'à titre d'orientation.

(e) Au Portugal, la léthalité a oscillé de 1950 à 1958 entre $1,1 \%$ et $14,5 \%$, d'après des données officielles $(2,3)$ et des informations complémentaires par le $\mathrm{D}^{\mathrm{r}}$ L. Cayolla da Motta. 
Le râclage de la muqueuse est un peu douloureux et donne lieu, par réflexe, à un certain larmoiement, mais on peut atténuer la douleur en faisant aspirer préalablement au malade, par le nez, un peu de solution de novocaïne à $2 \%$, ou bien en passant sur la muqueuse un tampon imbibé de ce même produit.

Forkner et Zia se sont limités à prélever la sécrétion nasale avec un tampon et à étendre le produit sur une lame. J'ai également essayé cette méthode, mais comme dès le début j'ai trouvé davantage de cas positifs par la méthode de prélèvement exposée ci-dessus, je l'ai ensuite utilisée systématiquement.

Une fois le produit obtenu, étendu sur la lame et coloré, on procède à l'examen microscopique avec objectif d'immersion, mais il est évident que le succès de la recherche dépend de la méticulosité et de la persistance apportées à l'examen de la lame.

Les parasites peuvent être facilement décelés ; dans quelques cas, en effet, soit immédiatement, soit après 5 ou 10 minutes, nous avions obtenu un résultat positif ; dans d'autres cas, cependant, ce n'est qu'après une longue période d'observation de la lame, qui d'ailleurs n'a jamais dépassé une heure, que nous avions trouvé des parasites. On peut trouver des Leishmanies dans n'importe quelle partie du frottis, mais c'est surtout dans les zones où il $\mathbf{y}$ a moins de globules de pus et moins de bactéries et où les globules rouges et blancs sont mieux conservés qu'ils se trouvent plus facilement et mieux conservés quant à leur morphologie ; c'est pourquoi que l'examen doit porter principalement sur ces zones.

Les Leishmanies conservent en général leur morphologie parfaitement normale (fig. 3) ; mais, parfois, nous avons trouvé quelques formes qui nous ont laissé des doutes quant à leur identification. On comprend qu'il en soit ainsi : soit par suite du contact, bien que fugace, des Leishmanies avec le mucus nasal, soit par l'action traumatique exercée au moment où l'on prépare le frottis. Il est certain que le diagnostic a toujours été fondé sur l'observation de Leishmanies absolument normales et dont l'identification n'offrait pas le moindre doute ; mais la découverte de Leishmanies modifiées dans leur morphologie ou même de formes suspectes nous a toujours conduits à un examen plus approfondi, jusqu'à ce que nous trouvions un parasite qui ne nous laissait aucun doute sur son identification. En général, les Leishmanies étaient extra-cellulaires, bien que, dans quelques cas, on les ait trouvées à l'intérieur des cellules ou dans des cellules en voie de désintégration.

La recherche directe des Leishmanies dans le sang s'est révélée positive dans quelques cas à l'examen direct. Ainsi, Henderson, 

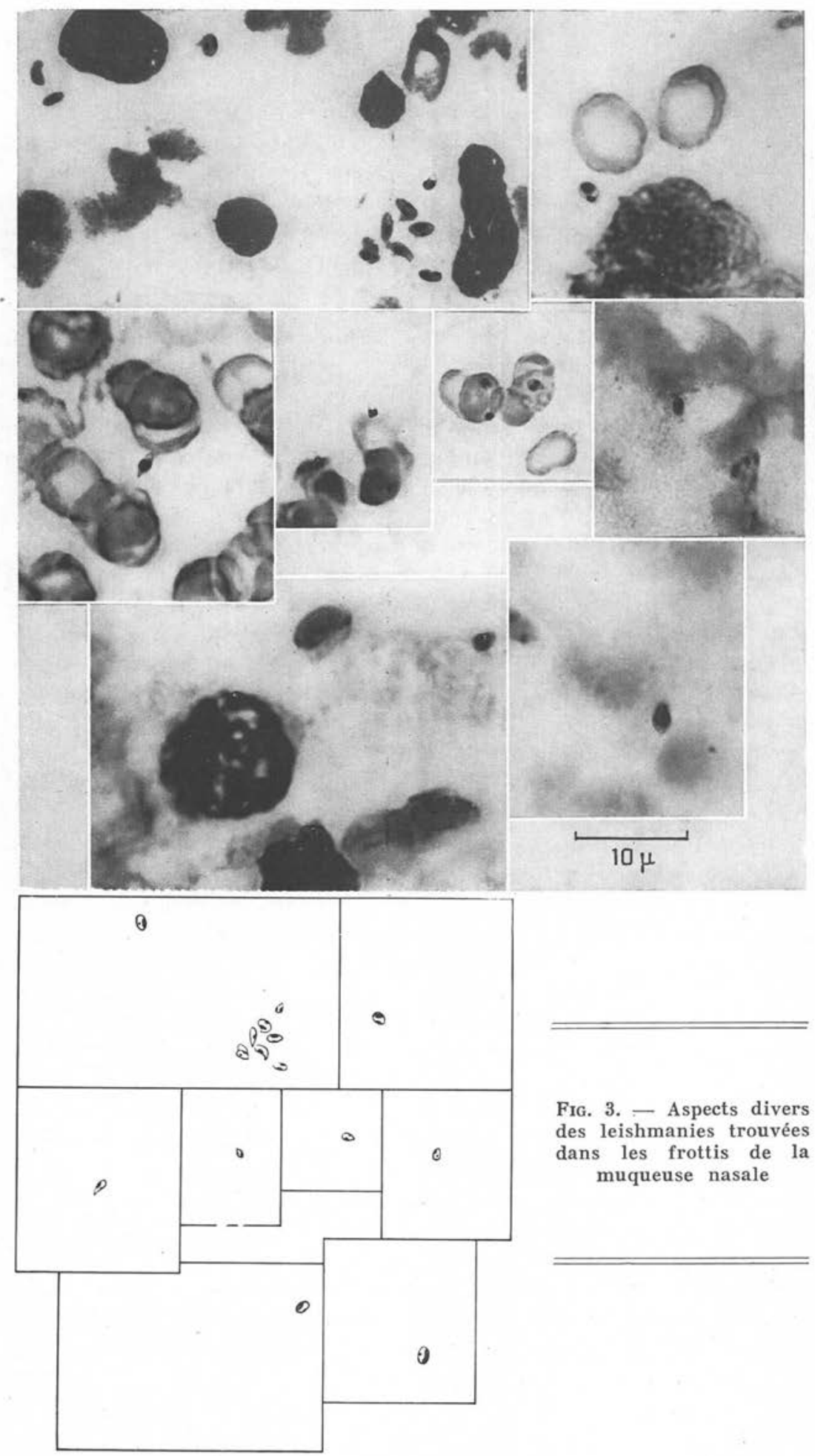

FIG. 3. - Aspects divers des leishmanies trouvées dans les frottis de la muqueuse nasale 
dans l'étude déjà citée, ne la signale comme positive que dans trois des cas, sur ses 300 malades, tandis que Marshall (27) a trouvé de rares Leishmanies dans le sang du même territoire dans 13 cas sur 15 examinés. La technique de cette méthode de recherche est simple, car il suffit de préparer des frottis de sang. Mais la rareté des cas positifs chez la plupart des auteurs ne la recommande pas comme méthode de routine.

L'hémoculture. - Suivant une technique analogue à celle qui était indiquée par Kolmer et coll. (28), nous avons prélevé, comme habituellement, $3 \mathrm{cc}$. de sang, dont nous avons semé $1 \mathrm{cc}$. directement en milieu de NNN dans un tube et $0,5 \mathrm{cc}$. dans un autre. Du sang restant, 1 cc. a été semé dans un tube contenant $20 \mathrm{cc}$. de solution de citrate de sodium à $1,5 \%$ en sérum physiologique, et $0,55 \mathrm{cc}$. dans un autre tube contenant le même volume de solution. Les deux tubes ont été laissés d'un jour à l'autre au frigorifique. Ainsi, après 24 heures, nous avons recueilli le sédiment de chaque tube avec une pipette et nous l'avons semé dans le milieu NNN. Par le passage du sang dans la solution de citrate de sodium, on propose d'éliminer les anticorps que le sang peut contenir et qui empêchent le développement des Leishmanies. Les tubes semés sont placés dans une étuve à $22-24^{\circ}$ et la recherche de Leptomones - les formes de culture des Leishmanies - est faite par l'examen d'une goutte de l'eau de condensation du milieu avec contraste de phase, sur fond noir, ou seulement avec le microscope optique normal.

Parmi nos résultats, le pourcentage le plus élevé de cas positifs a été constaté dans les cultures dont le sang a été passé préalablement par la solution de citrate ; on a constaté des résultats positifs qui ont varié entre 1 et 19 jours, comme il est indiqué ci-dessous :

\begin{tabular}{|c|c|c|c|}
\hline près & 1 & jour & \\
\hline Après & 3 & jours & \\
\hline Après & 4 & jours & $\ldots \ldots \ldots \ldots \ldots \ldots$ \\
\hline près & 6 & jours & $\ldots \ldots \ldots \ldots$ \\
\hline près & 9 & jours & 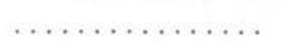 \\
\hline près & 10 & jours & $\ldots \ldots \ldots \ldots \ldots$ \\
\hline rès & 12 & ars & 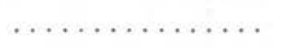 \\
\hline & 14 & Irs & $\ldots \ldots, \cdots, \cdots$ \\
\hline & & & \\
\hline
\end{tabular}

Kolmer et coll. signalent que les cultures se sont révélées positives habituellement entre 3 et 14 jours, mais qu'elles ne peuvent être considérées comme négatives avant 3 ou 4 semaines écoulées. 


\section{TABLEAU IV}

Résultats de la recherche de Leishmanies dans la muqueuse nasale et par hémoculture chez des malades atteints de Kala-azar

(de 1950 au 16-5.60)

\begin{tabular}{|c|c|c|c|c|c|c|c|c|c|c|c|c|c|c|}
\hline \multirow{4}{*}{ 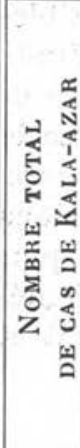 } & \multirow{2}{*}{\multicolumn{2}{|c|}{ 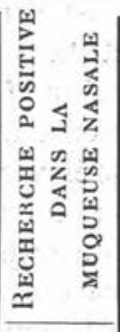 }} & \multicolumn{6}{|c|}{ HÉMOCULTURES POSITIVE } & \multirow{2}{*}{\multicolumn{2}{|c|}{ 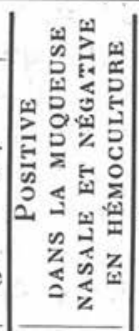 }} & \multirow{2}{*}{\multicolumn{2}{|c|}{ 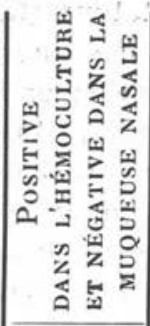 }} & \multirow[b]{2}{*}{ 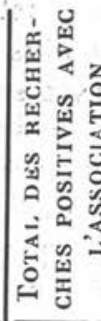 } & \multirow[b]{2}{*}{ 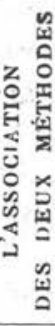 } \\
\hline & & & \multicolumn{2}{|c|}{ 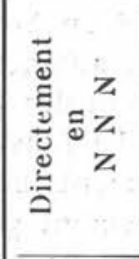 } & \multicolumn{2}{|c|}{$\begin{array}{c}\text { Par } \\
\text { passage } \\
\text { dans une } \\
\text { solution } \\
\text { de } \\
\text { citrate } \\
\text { de } \\
\text { sodium }\end{array}$} & \multicolumn{2}{|c|}{$\begin{array}{c}\text { Totaux } \\
\text { de la re- } \\
\text { cherche } \\
\text { directe } \\
\text { et } \\
\text { indirecte }\end{array}$} & & & & & & \\
\hline & \multicolumn{2}{|c|}{ Nombre: } & \multicolumn{2}{|c|}{ Nombres } & \multicolumn{2}{|c|}{ Nombres } & \multicolumn{2}{|c|}{ Nombres } & \multicolumn{2}{|c|}{ Nombres } & \multicolumn{2}{|c|}{ Nombres } & \multicolumn{2}{|c|}{ Nombres } \\
\hline & Absolus & $\%$ & Absolus: & $\%$ & Absolus & $\%$ & absolus & $\%$ & Absolus & $\%$ & Absolus & $\%$ & Absolus & $\%$ \\
\hline 38 & 6. & 16 & 4 & 10,5 & 13 & 34,2 & 13 & 34,2 & 3 & 7,8 & 10 & 26,3 & 16 & 42,1 \\
\hline
\end{tabular}

\section{Résultats}

Jusqu'à la présente date, notre casuistique comprend seulement 38 cas qui ont été réunis depuis 1950 et que nous devons à l'aimable attention du Prof. F. Fonseca et des $\mathrm{D}^{r s}$ Silva Nunes et Cordeiro Ferreira, à qui nous exprimons notre profonde gratitude. La rareté du Kala-azar dans les dernières années n'a pas permis d'obtenir une casuistique plus élevée, comme nous l'aurions voulu. Sur tous les malades, nous n'avons procédé qu'à un seul prélèvement, soit dans la muqueuse nasale, soit dans le sang.

Les résultats obtenus indiqués sur le tableau IV nous montrent qu'il a été possible de formuler le diagnostic étiologique du Kalaazar dans $16 \%$ des cas par la recherche des Leishmanies dans la muqueuse nasale $\left(^{*}\right)$ et dans $34,2 \%$ par l'hémoculture, avec un seul prélèvement. Le total des cas positifs par l'une et l'autre méthode de recherche a été de $42,1 \%$. Il est évident que si les prélèvements avaient été répétés, le pourcentage de cas positifs aurait certainement augmenté, car, dans quelques casuistiques relatives à la re-

(*) Le nombre de cas positifs pourra augmenter par inoculation du produit dans le hamster. Forkner et Zia (21). signalent trois malades où la recherche directe des leishmanies dans les sécrétions nasales a été négative et chez qui l'inoculation interpéritonéale dans le hamster a révélé des leishmanies. 
cherche dans la rate, les résultats ne se sont révélés positifs qu'à la $3^{*}$ et parfois à la $4^{*}$ ponction splénique, bien que, comme on le sait, les Leishmanies se localisent électivement dans cet organe.

$\mathrm{Si}$ nous comparons nos résultats avec ceux qui ont été obtenus par d'autres auteurs par la recherche dans la muqueuse nasale, nous constatons (tableau V) que le taux de positivité est sensiblement plus bas que celui qui a été constaté en Chine et dans l'Inde et assez supérieur à celui qui a été enregistré au Soudan. Cependant, je suis certain que le taux de positivité augmentera avec le perfectionnement de la recherche et la répétition des prélèvements. C'est ainsi que le nombre de cas où j'ai trouvé des Leishmanies dans la muqueuse nasale a été proportionnellement beaucoup plus élevé dans les dernières observations, certainement grâce à l'amélioration naturelle et instinctive des techniques de prélèvement et d'examen.

\section{TABlead $\nabla$}

Résumé comparatif de la recherche des Leishmanles dans le mucus nasal (les trols premières références) et dans la muqueuse nasale (dernlère référence) dans quelques régions du globe

\begin{tabular}{|c|c|c|c|}
\hline \multirow{2}{*}{ AUteur, date et pays } & \multicolumn{3}{|c|}{ RECHERCHES EFFECTUḰ:S } \\
\hline & Total & Positives & $\%$ positives \\
\hline $\begin{array}{l}\text { Forkner et Zia, Chine, } 1935 \\
\quad(21) \ldots \ldots \ldots \ldots \ldots \ldots \ldots \ldots \ldots \ldots \ldots\end{array}$ & 22 & 12 & 54,5 \\
\hline $\begin{array}{r}\text { Shortt et Swaminath, Inde, } \\
1935(24) \ldots \ldots \ldots \ldots \ldots \ldots \ldots\end{array}$ & 15 & 6 & 40 \\
\hline Henderson, Soudan, 1937 (19). & 120 & 9 & 7,5 \\
\hline $\begin{array}{r}\text { Fraga de Azevedo, Portugal, } \\
\quad 1960 \ldots \ldots \ldots \ldots \ldots \ldots \ldots\end{array}$ & 38 & 6 & 16 \\
\hline
\end{tabular}

\section{Conclusions}

Par un seul prélèvement dans la muqueuse nasale et par hémoculture, il a été possible de formuler sur des malades de Kala-azar le diagnostic étiologique, respectivement dans $16 \%$ et $34,2 \%$ des 
38 cas. Avec l'association des deux méthodes, le pourcentage de cas positifs a été de $42,1 \%$. Il est possible que ce pourcentage eût augmenté si les prélèvements avaient été répétés et si, pour l'hémoculture, l'on avait provoqué préalablement la spléno-contraction, ou bien si l'on avait administré, deux heures auparavant, 0,2 gramme de Néostibosan, comme le conseillent Napier et Gupta (29), pour augmenter les cas de positivité par la recherche de Leishmanies dans la rate.

Les avantages de ces méthodes de recherche par rapport à la ponction osseuse ou splénique sont évidents : plus grande simplicité, innocuité absolue, méthodes à la portée de tous.

Mais la recherche dans la muqueuse nasale a encore, quant à moi, un autre avantage. C'est qu'il est prouvé que les Leishmanies éliminées avec le mucus nasal peuvent conserver leur pouvoir pathogénique, tout au moins dans le Kala-azar chinois (21) ; c'est pourquoi, il est naturel d'admettre qu'il en est de même du Kalaazar méditerranéen. Il en résulte qu'il est possible que le malade atteint de Kala-azar soit contagieux, d'une manière identique à ce que l'on a constaté chez les animaux, ce qui peut expliquer les cas familiaux de la maladie, bien connus, même dans notre milieu, et les recrudescences épidémiques.

Quant à l'intérêt de la recherche des Leishmanies dans le sang, outre son importance pour le diagnostic, elle attire notre attention sur une particularité biologique du parasite : son passage fréquent par le sang, ce qui permet de comprendre le mécanisme possible de sa transmission par les Insectes hématophages. En outre, elle révèle le danger de la transmission des Leishmanies par transfusion, circonstance à considérer - évidemment, dans le cas des adultes seulement - , où la transfusion de sang d'un malade de Kala-azar (30) a donné lieu chez le récepteur à une forme cutanée.

Les données que je me suis permis de présenter ici nous permettent d'entrevoir une série de recherches qu'il y aurait intérêt à réaliser pour une meilleure connaissance des leishmanioses en général.

\section{RÉSUMÉ}

A la suite d'études identiques effectuées dans diverses régions du globe, l'auteur présente les résultats de la recherche de Leishmanies dans la muqueuse nasale et par hémoculture dans 38 cas de Kalaazar au Portugal.

Dans la recherche dans la muqueuse nasale, le pourcentage de cas positifs a été de $16 \%$, et par hémoculture de $34,2 \%$. L'application des deux méthodes a révélé $42,1 \%$ de cas positifs. 
Outre l'importance qu'elles présentent pour le diagnostic, les données ainsi obtenues se revêtent également du plus haut intérêt pour une meilleure connaissance de la biologie des Leishmanies et de l'épidémiologie des maladies auxquelles elles donnent lieu.

\section{BIBLIOGRAPHIE}

1. Fraga de Azevedo (J.), 1954. - L'état actuel du problème du Kala-azar au Portugal. Arch. Inst. Pasteur d'Algérie, 32, 3, 234-254.

2. Cayolla da Motta (L.), 1959. - Morbilidade e mortalidade das doenças infecciosas de notificação obrigatória em Portugal metropolitano (Continente e Ilhas Adjacentes) no decurso dos últimos $\mathbf{5}$ anos. (Morbidité et mortalité des maladies infectieuses à déclaration obligatoire sur le territoire portugais métropolitain (Continent et Iles Adjacentes), au cours des cinq dernières années) (1954-1958). Boletim dos Serv. Saúde Pública, Direcçâo Geral de Saúde, Lisbonne, 6, 313-487.

3. Castro Soares y Cayolla da Motta (L.), 1955. - Panorama das doenças infecto-contagiosas de notificação obrigatória, no ano de 1953 , em Portugal. (Aperçu sur les maladies infectio-contagieuses à déclaration obligatoire en 1953, au Portugal). Boletim dos Serv. Saúde Pública. Direcçâo Geral de Saúde, Lisbonne, 2, 101-134.

4. Leitâo (J. L. S.), 1953. - Leishmaniose canina em Lisboa e arredores. Aspecto clínico. Métodos de diagnóstico laboratorial. Lesões microscópicas. (La leishmaniose canine à Lisbonne et dans les environs. Aspect clinique. Méthodes de diagnostic en laboratoire. Lésions microscopiques). Arquivo Pat., Lisbonne, 25, 1-78.

5. Alvares (D.) et Pereira da Silva (E.), 1911. - Sobre a frequência do Kalaazar nos cães de Lisboa. (Sur la fréquence du Kala-azar chez les chiens de Lisbonne. Medic. Contemp., Lisbonne, 29, 13, 97.

6. Correia Mendes (A. B.), 1938. - Leishmaniose nos eães de Lisboa. (La leishmaniose chez les chiens de Lisbonne). Arq. Inst. Bact. Câmara Pestana, 7, 389-395.

7. Fraga de Azevedo (J.), Teixeira (G.) et Colto (A.), 1947. - Sobre a infestação por Leishmanias nos cães de Lisboa. (Sur l'infestation par les leishmanies des chiens de Lisbonne). Anais Inst. Med. Trop., Lisbonne, 4, 99-106.

8. LiPPI (M.), 1957. - La leishmaniosi viscerale e cutanea in Italia. Arch. Ital. Sci. Med. Trop. e Parassit., 38, 10, 503-527.

9. - - 1955. - New China's Achievements in the Treatment and Prevention of Kala-azar. Chinese Med. J. Peking, 73, 2, 91-99.

10. Fraga de Azevedo (J.), 1948. - Sobre a epidémiologia e diagnostico do Kala-azar. (Sur l'épidémiologie et le diagnostic du Kala-azar). Conferências de Pediatria, Lisbonne, 2, 115-171.

11. Corradetri (A.), 1954. - Lotta contro la leishmaniosi mediante la lotta contro i flebotomi in Italia. Rendiconti Istituto Superiore di Sanifá, Rome, 17, 5, 374-384.

12. NASIR-UD-DIN (M.), 1952. - D.D.T. in the Prevention of Kala-azar in East Pakistan. Pakistan J. of Health, 2, 1, 21-24. 
13. Almeida RoQue (R.), 1960. - Informação verbal dada amàvelmente, 1960. (Information verbale fournie aimablement en 1960).

14. TAvares (A.), 1943. - A Leishmaniose cutânea autoctone. (La Leishmaniose cutanée autochtone). Imprensa Médica, 9, 129-131.

15. Montin (X. V.), 1954. - Reflexiones a proposito de la diseminación de la leishmaniosis cutánea en España. Medicamenta, 21, 45-46.

16. Noronha (T.), 1959. - Mais quatro casos de Leishmaniose cutanea autoctone. (Encore quatre cas de Leishmariose cutanée autochtone). Arq. Pat., Lisbonne, 30, 105-128.

17. Fonseca (F.), Fraga de Azevedo (J.) e Marques da Gama (M.), 1956. - Nouveaux cas humains de Fascioliase hépatique au Portugal. Ann. Par. Hum. et Comp., 31, 1, 14-22.

18. DA Silva (J. R.) et De Paola (D.), 1956. - A punção-biopsia hepática no diagnostico do Calazar americano. (La ponction-biopsie hépatique dans le diagnostic du Kala-azar américain). Bol. Centr. Est. Hosp. Serv. Estado, Rio-de-Janeiro, 8, 1, 1-7, 1956.

19. Henderson (L. H.), 1937. - Clinical Observations on Kala-azar in the Fung Province of the Sudan. Trans. Roy. Soc. Trop. Med. a Hyg., 31, 179-190.

20. Forkner (C. E.) et $Z_{\text {IA }}$ (L. S.), 1934. - Viable Leishmania donovani in Nasa] and Oral Secretions of Patients with Kala-azar and the Bearing of this Finding on Transmission of the Disease. Journ. Exper. Med., 59, 491499.

21. Forkner (C. E.) et $Z_{\text {IA }}$ (L. S.), 1935. - Further Studies on Kala-azar. Leishmania in Nasal and Oral Secretions of Patients and the Bearing of this Finding on the Transmission of the Disease. Journ. Exper. Med., 61, 183-203.

22. Prata (A.) et Piva (N.), 1956. - Presença de Leishmania em pele aparentemente normal de pacientes com calazar. (Présence de Leishmania dans la peau apparemment normale de malades atteints de Kala-azar). Hospital, 49, 4, 481-486.

23. Bentley (C. A.), 1904. - Brit. Med. Journ., 2, 653-655.

24. Shortt (H. E.) et Swaminath (C. S.), 1935. - The Presence of Leishmania donovani in the Nasal Secretion of Cases of Indian Kala-azar. Ind. Journ. Med. Res., 23, 437-439.

25. Wu (Cheng-Chien), 1958. - Some Achievements on the study of Kala-azar in New China. Chinese Med. J. Peking, 77, 4, 307-309.

26. KIRK (R.), 1956. - Studies in Leishmaniasis in the Anglo-Egyptian Sudan. XII. Attempts to find a Reservoir Host. Trans. Roy. Soc. Trop. Med. et Hyg., 50, 2, 169-177.

27. Marshall (W. E.), 1911. - Kala-azar Commission to investigate the Prevalence and the cause of the Disease in the Eastern Sudan (2). Pathological Report. Wellcome Trop. Res. Lab., $4^{\text {th }}$ Report, 157.

28. Kolmer (J. A.), Spaulding (E. H.) et Robinson (H. W.), 1952. - Approved Laboratory Technic., London:

29. NAPIER (L. E.) et Gupta (C. R.), 1930, - The value of a Provocative Dose of Pentavalent Antimony in the Diagnosis of Kala-azar. Indian. J. Med. Res., 17, 3, 749-753.

30. André (R.), Brumpt (L.), Dreyfus (B.), Penelece (A.) et Jacob (S.), 1957. Leishmaniose cutanée, leishmaniose cutanéo-ganglionnaire et Kala-azar transfusionnel. Bull. et Mém. Soc. Méd. Hôp. de Paris, 25-26, 854-860.

Institut de Médecine tropicale, Lisbonne (Portugal) 